\title{
Design of mini wind tunnel based on coanda effect
}

\author{
Yassen El-Sayed Yassen, Ahmed Sharaf Abdelhamed \\ Mechanical Power Engineering, Faculty of Engineering, Port Said University, Port Said, Egypt \\ Email address: \\ y_yassen70@yahoo.com (Y. El-S. Yassen),sh_ahmed99@yahoo.com (A. S. Abdelhamed)
}

\section{To cite this article:}

Yassen El-Sayed Yassen, Ahmed Sharaf Abdelhamed. Design of Mini Wind Tunnel Based on Coanda Effect. American Journal of Aerospace Engineering. Special Issue: Hands-on Learning Technique for Multidisciplinary Engineering Education. Vol. 2, No. 1-1, 2015, pp. 31-37. doi: 10.11648/j.ajae.s.2015020101.13

\begin{abstract}
An experimental investigation and CFD treatment were employed to design mini-wind tunnel based on Coanda effect for model tests and basic research. The inlet source flow is efficiently creating smooth steady airflow with acceptable noise, achieving the possibility of placing the test target closer to the source of flow with reasonable estimates of turbulence intensity. The design aims at achieving flow uniformity in the working section midplane, preventing separation in the contraction and minimizing the boundary-layer thickness. Intensive measurements after construction demonstrate the significance of the design process and validate the CFD predictions. The results are represented in graphic form to indicate the aspects of the contraction ratio. The numerical and experimental results show the uniformity of velocity distribution inside the working section. Tracing of separation and backflow is crucial allowing a variety of realistic demonstrations to be performed. The numerical solution provides a powerful tool to demonstrate the rate of boundary-layer growth inside the working section and validate against the empirical correlations with insignificant wall-friction drag. Assessment study to address large-scale wind tunnel based on coanda effect would be considered.
\end{abstract}

Keywords: Separation, CFD, Coanda Effect, Mini-Wind-Tunnel, Boundary-Layer Growth

\section{Introduction}

A conventional wind-tunnel design is a complex field involving many fluid mechanics and engineering aspects. The first attempt in providing some guidelines for the complete design of low-speed wind tunnels was that due to [1]. However, recent experimental studies of flow through individual components of a wind tunnel [2-4] have led to increase understanding and design philosophy for most of the components of wind tunnel. More theoretical and experimental investigations have been written about this topic and e.g. [5-8] are useful references when designing and constructing conventional low-speed wind-tunnels. Typically, the air is moved through conventional tunnel using a fan or blower. The airflow created by the fan entering the tunnel is itself highly turbulent due to the fan blade's motion. The air moving through the tunnel needs to be relatively turbulence-free [9]. Therefore, the overall length of the tunnel increases to smooth out the turbulent airflow before reaching the subject of the testing. This design is less than ideal for a wind tunnel but it is still the prevalent design.

In the present study, both of computational treatment based on RNG turbulence model and experimental measurements are implemented to design an unconventional wind-tunnel (mini-wind tunnel) based on Coanda effect for model tests and laboratory teaching purpose. The inlet source flow is efficiently creating smooth steady airflow with acceptable noise, achieving the possibility of placing the test target closer to the source of flow with reasonable estimates of turbulence intensity. The design aims at achieving the flow uniformity in the working section midplane, without separation in the contraction and minimizing the boundary-layer thickness at entrance to the working section. Calibration of the proposed mini wind tunnel after construction is carried out. The boundary-layer growth inside the working section is determined using the empirical correlations and validated against the numerical results. Wall-friction drag is estimated. Both of CFD predictions and experimental results are validated against the uniformity of velocity distribution inside the working section. Also, tracing of separation and backflow through the tunnel is carried out for different values of contraction ratios. The overall length of mini wind tunnel is $160 \mathrm{~cm}$ with contraction ratio of 2.82 and the cross-sectional area of test section is $19 \times 19 \mathrm{~cm}^{2}$ with length $50 \mathrm{~cm}$. 


\section{Experimental Design}

\subsection{Mechanism of Supply Air}

The mechanism of air flow as shown in Fig.1 may be demonstrated in several steps which can all be explained by the Coanda effect, the Venturi effect, and Bernoulli's principle. First, the air is drawn in through the base of the machine, powered by what is called a mixed-flow impeller of 38 watt. It has nine fins with rows of tiny holes that reduce the friction caused by colliding high and low air pressure. The air is accelerated through an annular aperture and then passed over an airfoil-shaped ramp that channels (36 slits) its direction. By propelling air out of a $1.6 \mathrm{~mm}$ slit located on the inside of its ring, air flows across one side of the airfoil, the Coanda surface. This results in entrainment of surrounding air, just downstream of the airfoil. The Venturi effect is a factor in creating the optimal air velocity, resulting in the Coanda effect As seen in the cross section of Fig.1, as the air exits the airfoil, it is funneled through a small slit. The fluid velocity greatly increases with this reduction in area, resulting in optimal entrainment of air. Also, the air is drawn in from behind, or induced, and this can be explained by Bernoulli's principle. As the air leaves the airfoil at a higher velocity than the surrounding air, it creates an area of low pressure. This pressure differential between the high velocity air and still air behind is what draws in the induced air. This combination of entrainment from the Coanda effect and inducement by Bernoulli's principle is what makes the air flow 15 times its air intake and an average turbulence level $0.024 \%$. According to the above explanation, the optimal Coanda surface profile for entrainment of air has found, one with an airfoil cross section with a $14^{\circ}$ angle between the top and bottom surfaces and whose cord length is constant at approximately $10 \mathrm{~cm}$. The construction of the airfoil was done by using fiberglass and a foam exoskeleton as can be seen in Fig.1.

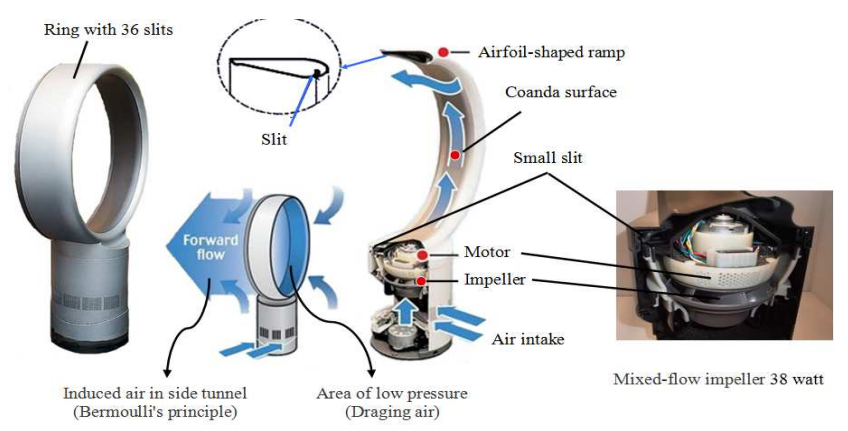

Fig 1. Air supply and construction of the airfoil.

\subsection{Mini-Wind Tunnel Components}

Manufacturing a mini-wind tunnel of card-board that was covered inside and outside with specific foil paper so as to obtain smooth surface of the inside tunnel. The general layout of the mini wind tunnel is shown in Fig. 2.

A mini-wind tunnel is manufactured as an open-circuit rig powered by a 38 watt induction motor with low average turbulence intensity $0.024 \%$ and low average noise source level $(21.5 \mathrm{~dB})$ compared with the allowable acoustics noise level in conventional small wind tunnels (70-90) $\mathrm{dB}[10,11]$. The flow is accelerated by contraction (contraction ratio, CR $=2.82$ ) into a $19 \mathrm{~cm}$ square test section. The dimensions and specifications of the mini wind tunnel are shown in Fig. 3 and Table (1), respectively.

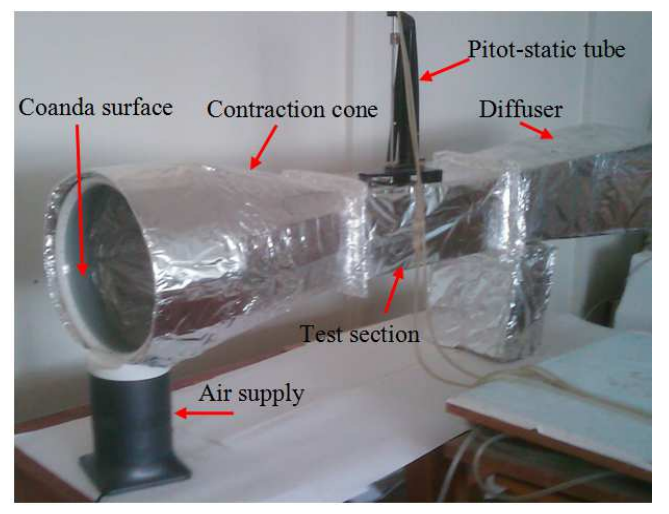

Fig 2. Test rig of mini-wind tunnel.

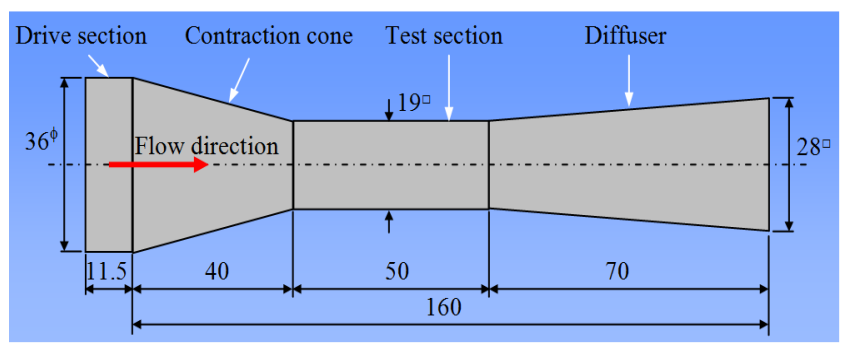

Fig 3. Mini-wind tunnel, dimensions in (cm).

Table 1. Specifications of Mini-wind tunnel.

\begin{tabular}{ll}
\hline Overall length $(\mathrm{cm})$ & 160 \\
Entrance cone $(\mathrm{cm})$ & $36^{\phi}$ \\
Contraction ratio & 2.82 \\
Test section $(\mathrm{cm})$ & $19 \times 19 \times 50$ \\
Average turbulence leve at inlet tunnel & $0.024 \%$ \\
Air mass flow rate at inlet tunnel & 0.008 to $0.0182 \mathrm{~kg} / \mathrm{s}$ \\
Average noise source level at inlet tunnel & $21.5 \mathrm{~dB}$ \\
Drive (motor) & 38 watt \\
\hline
\end{tabular}

\subsection{Experimental Measurements}

The velocity profile through the mini-wind tunnel at eight sections (two in contraction section, four in test section, and two in diffuser section) is measured using Static-Pitot tube of $6 \mathrm{~mm}$ diameter. A simple slide probe traversing mechanism is used to locate the probe tip at every location (12 points, and $1.5 \mathrm{~cm}$ between each two successive points and five points, 5 $\mathrm{mm}$ between each near the wall). The collected measurements data at measuring station locations were averaged and used to calculate the mean velocity distribution of the air flow through the mini-wind tunnel. The uncertainty, using root-sum-square method in the measured mean velocity is $\pm 4.8 \%$ within $95 \%$ confidence. The measurement sections through the mini-wind tunnel (half of the tunnel) are shown in Figs. $(4,5)$. 


\section{Mesh-Independency}

The quality of the grid plays a crucial role in the accuracy of CFD simulations and for achieving a fully converged solution. The body-fitted grid has the flexibility to model problems with irregular geometries. It offers modeling mini wind tunnel geometry accurately and produces fine grid resolution in regions of special interest. One of the important tasks in grid generation is to obtain grid-independent results and it is highly necessary to have a flow passage which is represented by as many grid points as necessary to give a realistic flow. The domain is meshed using an unstructured grid with different densities are tested. The refinement mesh with a factor of 2 is chosen around the airfoil for stability. However, the mesh of the final design shown in Fig. 6 has 61784 cells and average skew factor of 0.3481 produced best results.

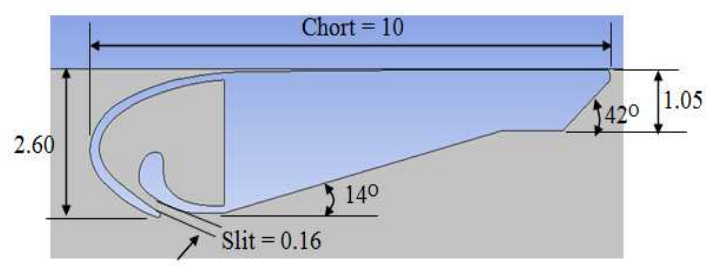

Fig. 4. Airfoil, dimensions in (cm)

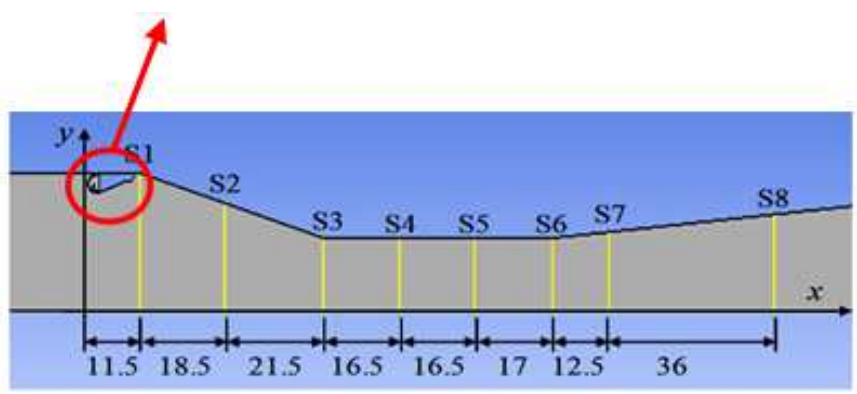

Fig. 5. Measurement Locations, dimensions in (cm).

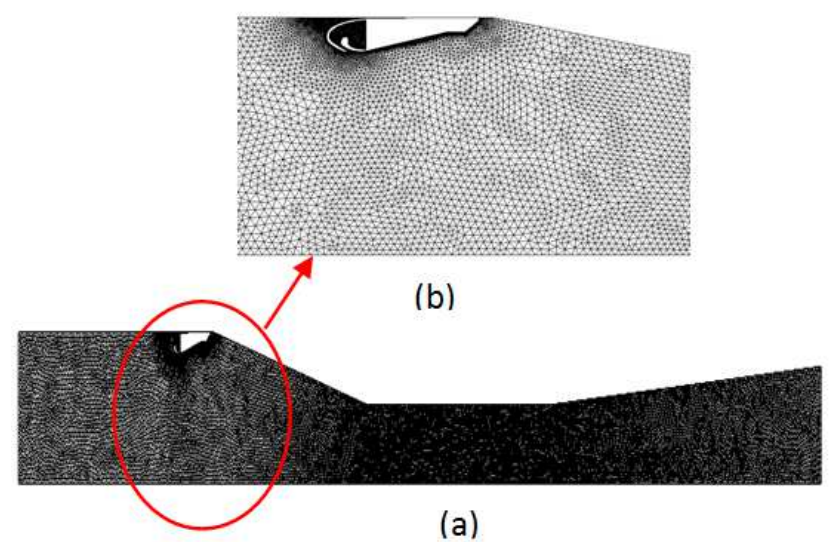

Fig. 6. (a) Grid construction (b) Grid details around the airfoil.

\section{Results and Discussion}

When investigating the results of the design and construction of an unconventional small-scale laboratory wind tunnel (mini-wind tunnel) based on Coanda. Effect, there are several key factors that have to be checked carefully. The most important factors concern mean-flow variations in time and space over the test-sectional cross section area, the turbulence fluctuation intensities, both in the streamwise and cross-stream directions. CFD simulation is performed using ANSYS FLUENT 14 in order to handle the axi-symmetric steady incompressible turbulent air flow using RNG turbulence model with enhanced wall treatment. In the present study, different contraction ratios $(1.63,2.54,2.82,3.14$, and 5.19) are chosen for mini-wind tunnel based on Coanda effect with inlet mass flow rate $0.0182 \mathrm{~kg} / \mathrm{s}$, inlet diameter of the tunnel $0.36 \mathrm{~m}$, and constant angle of the diffuser $3.5^{\circ}$. The inlet boundary conditions used in numerical solution are exactly the same as experiments.

Figure 7(a) shows the predictive results, for streamwise velocity profile at eight successive stations for contraction ratio $\mathrm{CR}=1.63$. It can be seen that the velocity is maximum near the wall in sections 1 and 2 due to Venturi effect. The inlet velocity to the test section (section 3 ) is non-uniform, and the velocity profiles at sections 4,5 , and 6 are uniform. Also, the value of velocity in the test section is less than $2 \mathrm{~m} / \mathrm{s}$. However, it can be observed that no separation or back flow in the contraction cone, as shown in Figs. 7(b, c).

Figure 8(a) illustrates the numerical results for streamwise velocity profile at eight successive stations for contraction ratio $\mathrm{CR}=2.54$. It can be noticed that the maximum velocity takes place near the wall in sections 1 and 2 . The inlet velocity profile to test section (section 3 ) is close to uniformity but the velocity profiles at sections 4,5 , and 6 are uniform in shape and approaching $2 \mathrm{~m} / \mathrm{s}$ in the test section compared with the case where $\mathrm{CR}=1.63$, Fig. 7(a). Moreover, no separation and back flow in the contraction cone, as shown in Figs. 8(b, c).

Figure 9(a) shows the predictive results for streamwise velocity profile at eight successive stations for contraction ratio $\mathrm{CR}=2.82$. It is clear that the velocity is maximum near the wall in sections 1 and 2 . The inlet velocity to test section (section 3) is almost in uniform profile. While the velocity profiles at sections 4,5 , and 6 are uniform with value greater than $2 \mathrm{~m} / \mathrm{s}$ in the test section compared with the cases where $\mathrm{CR}=1.63$ and 2.54, as shown in Figs. (7.a, 8.a), respectively. Also, it can be seen that there is no separation and back flow in the contraction cone, as shown in Figs. 9 (b, c).

The predictive results for streamwise velocity profiles at eight successive stations for contraction ratio $\mathrm{CR}=3.14$ are shown in Fig.10(a). The maximum velocity takes place near the wall in sections 1 and 2. It can be observed that the flow seems to be irresistible for separation as shown in Figs. 10(b, c). This is attributed to the effect of contraction ratio $(C R=3.14)$. However, the velocity profile at the inlet to test section (section 3) is nearly uniform while the velocity profiles at sections 4,5 , and 6 are uniform with value approaching $2 \mathrm{~m} / \mathrm{s}$ in the test section compared with the other cases, $\mathrm{CR}=1.63,2.54$, and 2.82 .

For contraction ratio $=5.19$, the flow suffers from severe separation and back flow in the contraction cone, as shown in Figs. 11(a,b,c). The effect of separation is quite clear on the 
value of the velocity in test section that is less than $2 \mathrm{~m} / \mathrm{s}$. However, flow separation should be avoided inside the tunnel circuit to prevent flow unsteadiness and associated noise as well as to minimize losses.

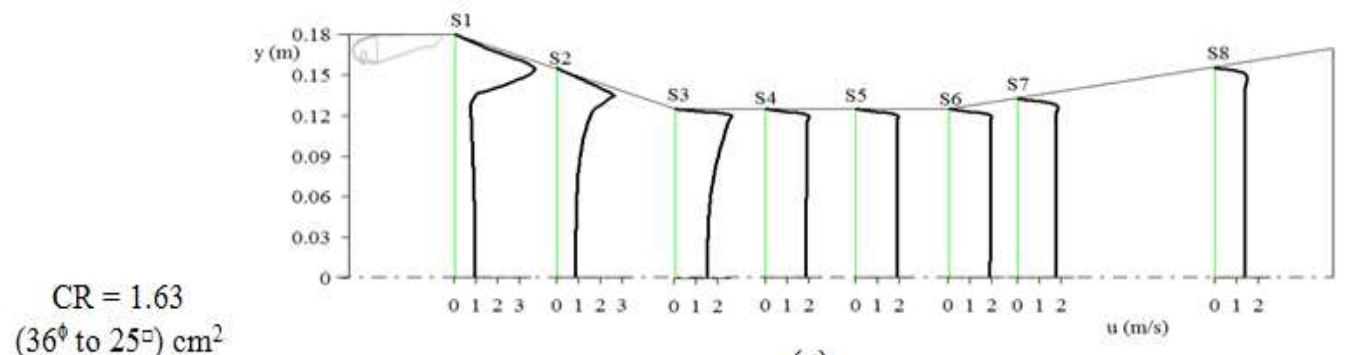

(a)

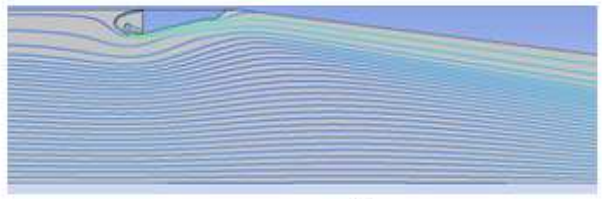

(b)

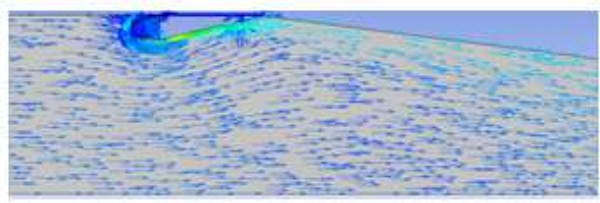

(c)

Fig 7. (a) Velocity profiles (b) Streamlines (c) Velocity vectors for $C R=1.63$.

$\mathrm{CR}=2.54$

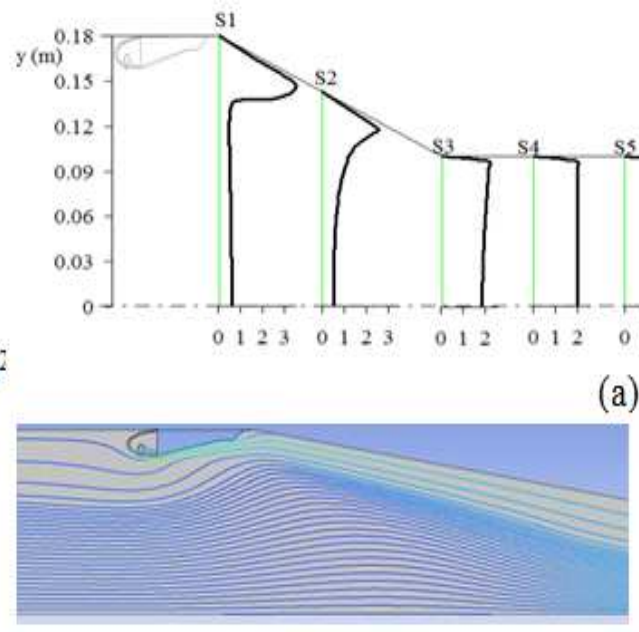

(b)

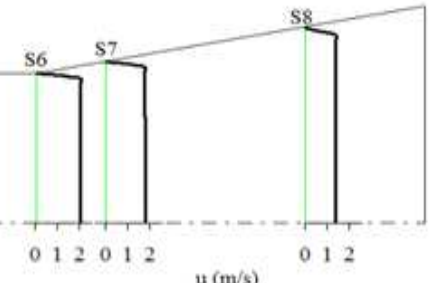

a)

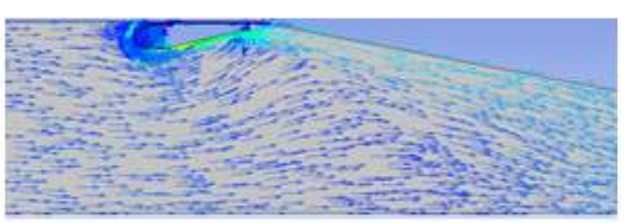

(c)

Fig 8. (a) Velocity profiles (b) Streamlines (c) Velocity vectors for $C R=2.54$.

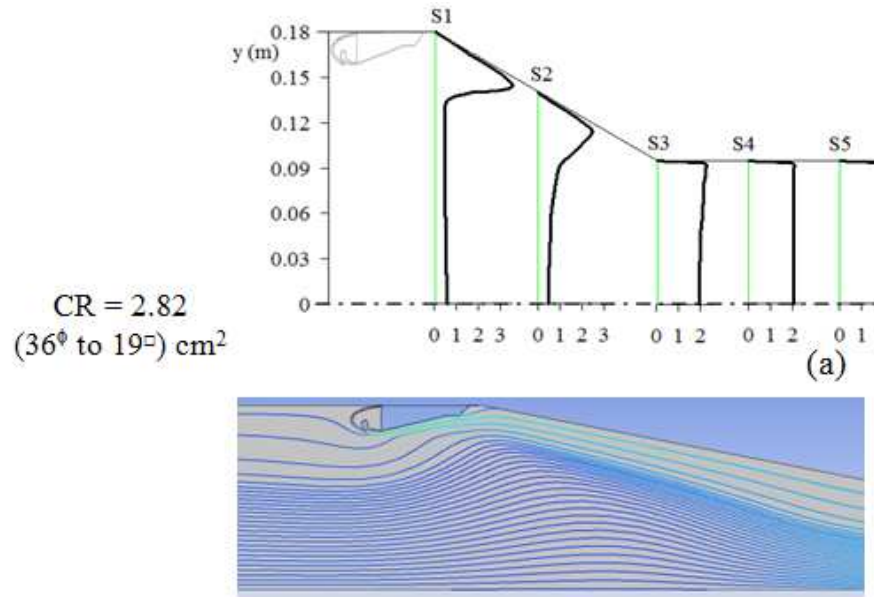

(b) (c)

Fig 9. (a) Velocity profiles (b) Streamlines (c) Velocity vectors for $C R=2.82$. 


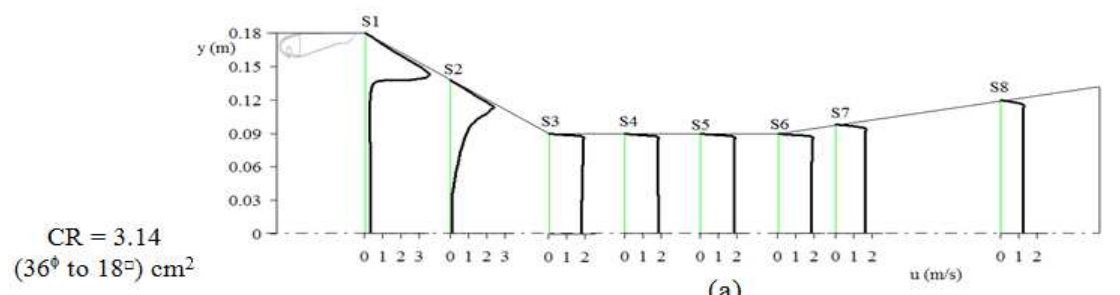

(a)

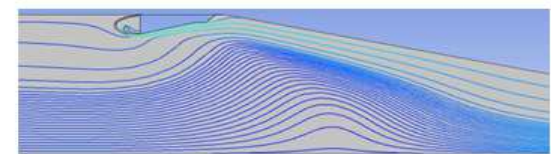

(b)

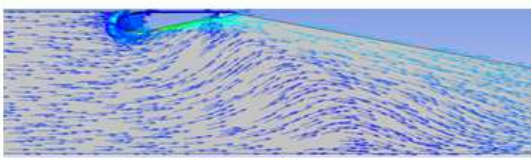

(c)

Fig 10. (a) Velocity profiles (b) Streamlines (c) Velocity vectors for $C R=3.14$.

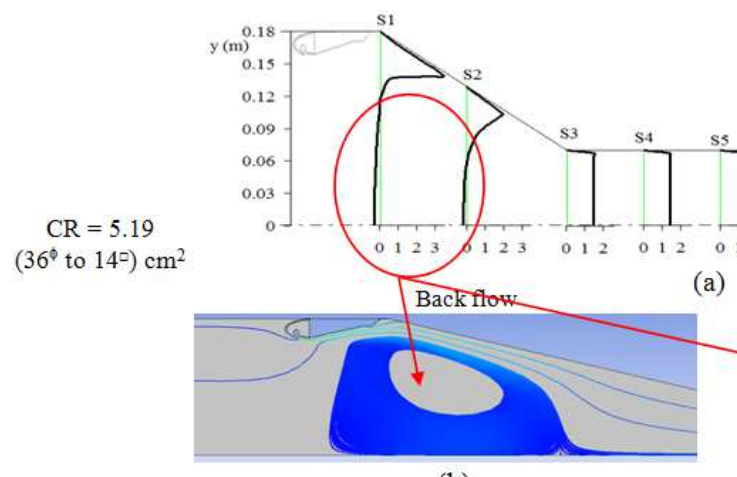

(b) (a)

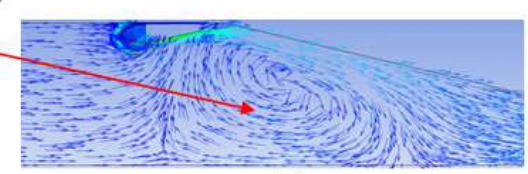

(c)

Fig 11. (a) Velocity profiles (b) Streamlines (c) Velocity vectors for CR $=5.19$.
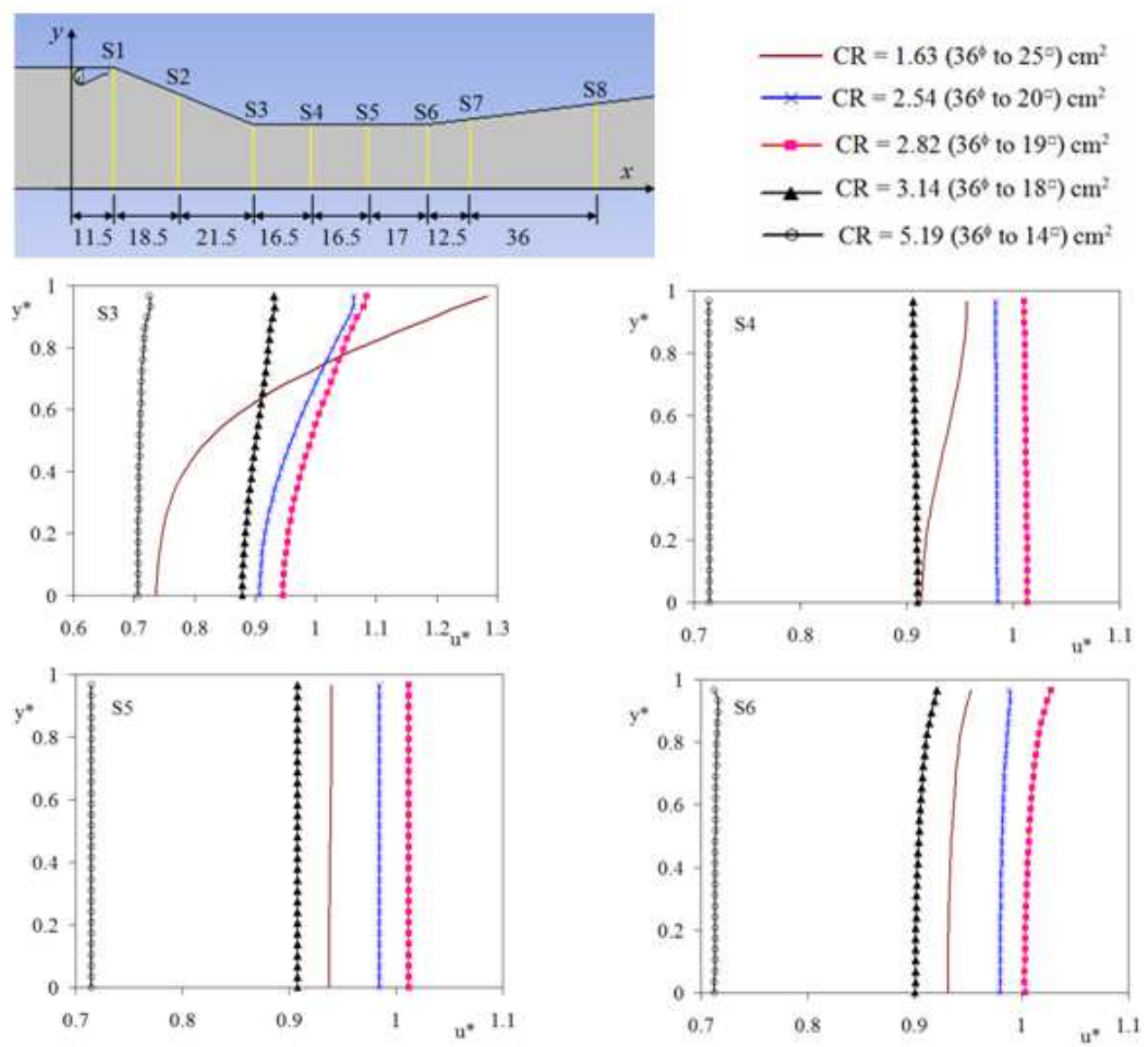

Fig 12. Velocity profile over working section for different contraction ratios. 
The working section represents one of the important parts of the tunnel. An intensive numerical investigation is carried out in order to demonstrate the influence of contraction ratio on the velocity profile inside the test section. Figure 12 shows the predictive results for streamwise velocity profile over working section (sections 3, 4, 5, and 6) for different contraction ratios $(1.63,2.54,2.82,3.14$, and 5.19). It can be noticed the significance of $\mathrm{CR}=2.82$ that achieves the uniformity inside the test section and high velocity value compared with the other cases of contraction ratios.

One of the most important aspects of the flow quality in a wind-tunnel is the level of turbulence intensity. Figure 13 shows the numerical results of turbulence intensity at inlet to the mini-wind tunnel for different contraction ratios. The contraction ratios $(2.54,2.82$, and 3.14$)$ demonstrated relatively reasonable estimates of turbulence intensity $0.024 \%$. Comparison of the numerical values of turbulence intensity, $\mathrm{CR}=2.82$ appeared to have a more uniform velocity profile, no separation and back flow, and hence it was selected for manufacture.

However, the performance of the chosen mini-wind tunnel with contraction ratio $(\mathrm{CR}=2.82)$ still requires testing after construction, to validate the CFD simulations. The inlet contraction plays a critical role in determining the flow quality in the test section. The contraction accelerates and aligns the flow into the test section. The size and shape of the contraction dictate the final turbulence intensity levels in the test section. The contraction stretches vortex filaments, which reduces axial but intensifies lateral turbulent fluctuations. The length of the contraction should be sufficiently small to minimize boundary-layer growth and manufacturing cost but long enough to prevent large adverse pressure gradients along the wall, generated by streamline curvature, which can lead to flow separation. More comprehensive experimental data are required to validate the numerical solution. Figure 14 shows the velocity profiles for the numerical results and measurements at eight measuring sections for $\mathrm{CR}=2.82$. It can be seen that the numerical solution based on RNG turbulence model is able to represent the experimental measurements. Fair agreement between the predictive results and experimental data is achieved.

Assuming that the rate of boundary layer growth starts at the working section $(0.19 \times 0.19) \mathrm{m}^{2}$, both of Blasius and Von Karman Integral formulae are used to estimate the laminar boundary layer characteristics as shown in table (2). It is noticed that the wall friction drag is minimum.

Figure15 shows the boundary-layer growth based on the numerical solution (blue color) and an empirical correlation over the working section. The predicted results provide a powerful tool to demonstrate the rate of boundary-layer growth inside the working section and validate against the empirical correlations (Blasius and Karman Integral formulae).

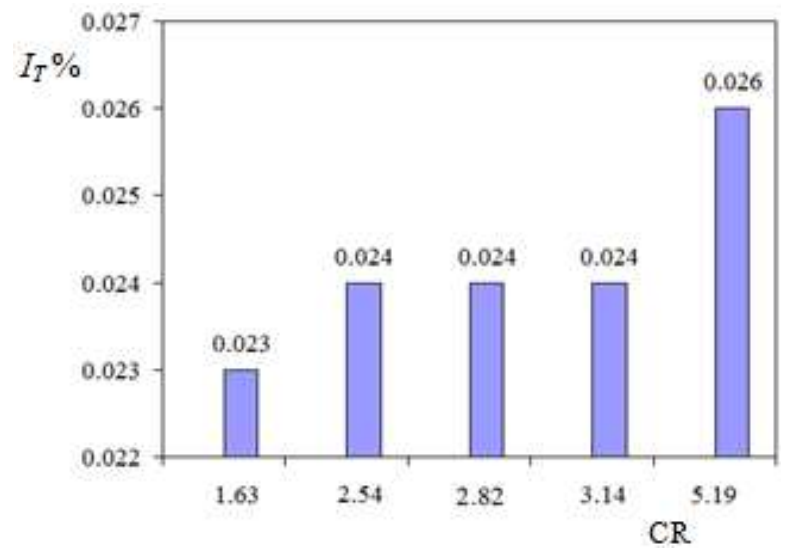

Fig 13. Turbulent intensity for different contraction ratios.

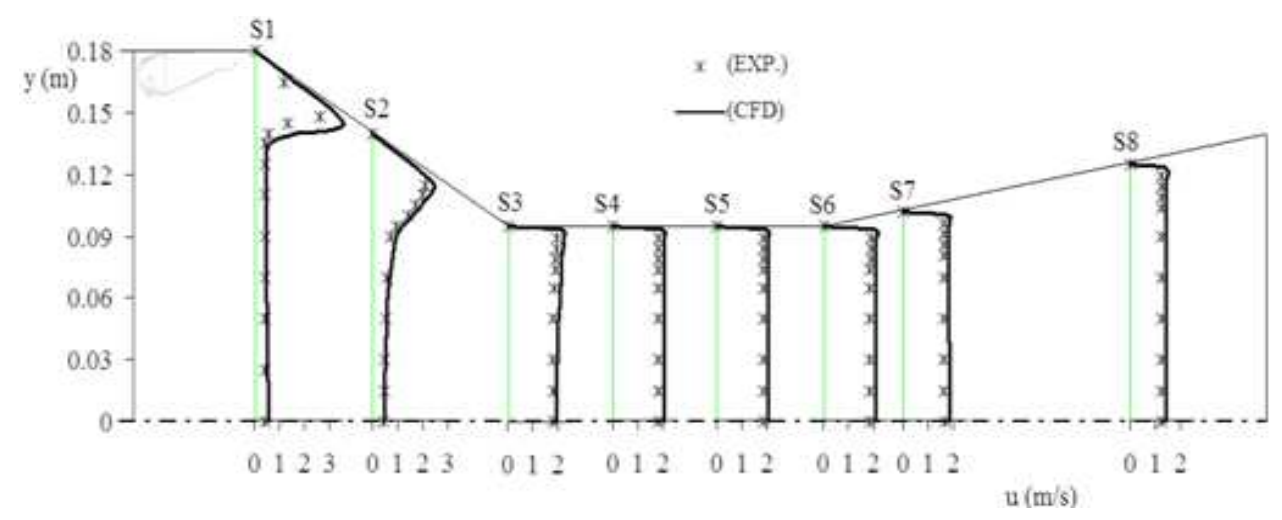

Fig 14. Velocity profiles at eight sections for $C R=2.82$.

Table 2. Laminar Boundary-layer characteristics $(x=0.5 \mathrm{~m}$ and Rex $=6.5 x 104)$.

\begin{tabular}{lll}
\hline & Blasius & ${\text { Von Karman Integral (parabolic profile) } \boldsymbol{u}=\boldsymbol{\alpha} \boldsymbol{y}+\boldsymbol{\beta} \boldsymbol{y}^{\mathbf{2}}}$ \\
\hline Boundary-layer thickness $\delta(\mathrm{mm})$ & 9.71 & 10.72 \\
Displacement thickness $\delta^{*}(\mathrm{~mm})$ & 3.39 & 3.56 \\
Momentum thickness $\theta(\mathrm{mm})$ & 1.30 & 1.34 \\
Skin-friction coefficient $\mathrm{C}_{\mathrm{f}}$ & $2.6 \times 10^{-3}$ & $2.86 \times 10^{-3}$ \\
Wall-shear stress $\tau_{\mathrm{w}}\left(\mid \mathrm{N} / \mathrm{m}^{2}\right)$ & $6.39 \times 10^{-3}$ & $7.08 \times 10^{-3}$ \\
Wall-friction Drag, D $(\mathrm{N})$ & $0.61 \times 10^{-3}$ & $0.67 \times 10^{-3}$ \\
\hline
\end{tabular}




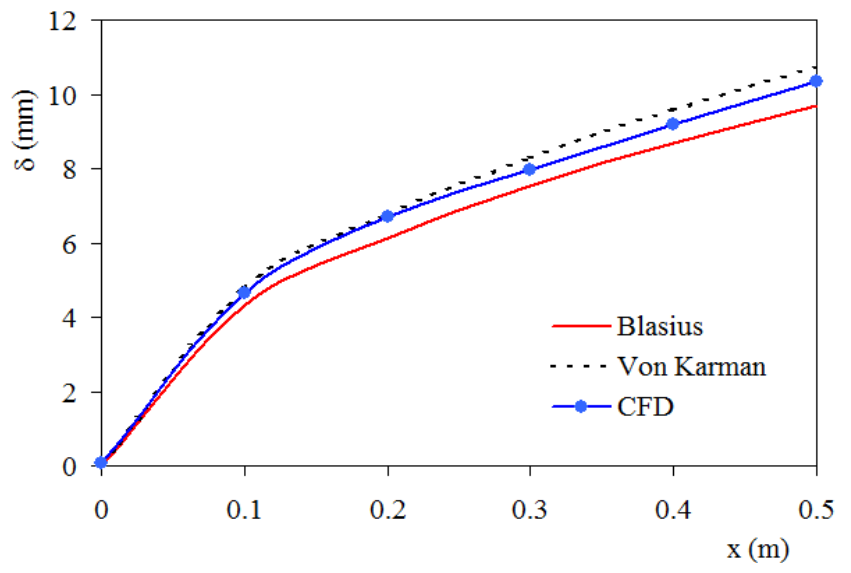

Fig 15. Boundary-layer growth inside the working section.

\section{Conclusions}

Computational treatment based on RNG turbulence model and experimental measurements are implemented to design an unconventional small-scale laboratory wind tunnel (mini-wind tunnel) based on Coanda effect suitable for laboratory model tests and basic research. Different contraction ratios are tested against uniformity in the working section and characteristics of the flow. Care must be taken to ensure no flow separation occurs in the ducts, which may also require acoustic treatment to minimize flow noise. Clearly, uniform velocity in the test section is desired as well as no separation, no back flow and minimum noise with relatively reasonable estimates of turbulence intensity. More comprehensive experimental measurements are carried out to validate the numerical solution for chosen contraction ratio. The predicted results provide a powerful tool to demonstrate the rate of boundary-layer growth inside the working section and validate against the empirical correlations with insignificant wall-friction drag. Assessment study to address the conventional wind tunnel would be considered. A significant challenge therefore exists to develop accurate and efficient design tools for flow modeling of large-scale wind tunnel in three dimensions based on Coanda effect.

\section{Nomenclature}

$\mathrm{I}_{\mathrm{T}} \quad$ Turbulent intensity

$\operatorname{Re}_{\mathrm{x}} \quad$ Local Reynolds number from the working section $\mathrm{u} \quad$ Velocity in $\mathrm{x}$ direction, $\mathrm{m} / \mathrm{s}$

$\mathrm{U}$ Mean velocity at the inlet of the working section, $\mathrm{m} / \mathrm{s}$

$\mathrm{u}^{*} \quad$ Dimensionless velocity, $=\mathrm{u} / \mathrm{U}$

$x \quad$ Axial distance from the entrance of the working section, $\mathrm{m}$

y Normal distance to wall, $\mathrm{m}$

$\mathrm{y}_{\max } \quad$ Maximum normal distance at each section, $\mathrm{m}$

$\mathrm{y}^{*} \quad$ Non-dimensional normal distance, $\mathrm{y} / \mathrm{y}_{\max }$

\section{References}

[1] Bradshaw, P., and Pankhurst, R. C., "The design of low-speed wind tunnels", Progress in aeronautical sciences 6, $1-69$, 1964.

[2] Mehta, R.D., "Aspects of the Design and Performance of Blower Wind Tunnels Components", Ph.D. Thesis, Department of Aeronautics, Imperial College, University of London, 1978.

[3] Mehta, R.D. and Bradshaw, P., "Design Rules for Small Low Speed Wind Tunnels", Aeronaut. J., Vol. 83, No. 827, pp. 443-449, 1979.

[4] Bell, J.H., and Mehta, R.D., " Contraction Design for Small Law-Speed Wind Tunnels", National Aeronautics and space, CA 94305, 1988.

[5] Rae, W. H., and Pope, A., "Low-speed wind tunnel testing", 2nd edn. John Wiley and sons, 1984.

[6] Mansi, S., Neha, S., and Sunil, K. Y., "Review of Design and Construction of an Open Circuit Low Speed Wind Tunnel", Global J. of Researches in Eing. (A), V. XIII Issue V Version I, 2013.

[7] Björn L., and Arne V. J., "Design and Evaluation of a Low-Speed Wind-Tunnel with Expanding Corners", Technical Reports from Royal Institute of Technology, Department of Mechanics, SE-100 44 Stockholm, Sweden, 2002.

[8] Seidel, M., "Construction, design, manufacturing, and calibration of the German-Dutch wind tunnel (DNW)", Tech. Rep., Duits-Nederlandese Windtunnel (DNW), 1982.

[9] Goldstein, E.,"Wind Tunnels, Don't Count Them Out," Aerospace America, Vol. 48 4, pp. 38-43, 2010.

[10] Salam, C.A., and Ali, M.A., "Design and Fabrication of A Bench Mounted Closed Loop Wind Tunnel", MIST J. of Science and technology, Vol. 2, No. 1, 2010.

[11] Wagner, S., Bareiß, R. and Guidati, G, "Wind Turbine Noise", Springer-Verlag, Berlin, pp. 14-21, 1996. 